\title{
Spectral control of broadband light through random media by wavefront shaping
}

\author{
Eran Small ${ }^{1}$, Ori Katz ${ }^{1}$, Yefeng Guan ${ }^{1,2}$, and Yaron Silberberg ${ }^{1}$ \\ ${ }^{1}$ Department of Physics of Complex Systems, The Weizmann Institute of Science, Rehovot 76100, Israel \\ ${ }^{2}$ State Key Laboratory of Optoelectronic Materials and Technologies, Sun Yat-sen University, Guangzhou 510275, China
}

Compiled November 2, 2018

A random medium can serve as a controllable arbitrary spectral filter with spectral resolution determined by the inverse of the interaction time of the light in the medium. We use wavefront shaping to implement an arbitrary spectral response at a particular point in the scattered field. We experimentally demonstrate this technique by selecting either a narrow band or dual bands with a width of $5.5 \mathrm{~nm}$ each.

OCIS codes: (030.6140) Speckle; (070.4790) Spectrum analysis; (320.5540) Pulse shaping.

The propagation of light in disordered media results in scattering, which is often a major limitation in many optical applications, ranging from microscopy to astronomy. However, optical wave propagation through disordered media can be controlled and even inverted using wavefront-shaping [1] or phase-conjugation [2], a result that has been extensively studied in time-reversal techniques in acoustics and radio-frequency electromagnetic waves 3, 4. Perhaps surprisingly, random scattering can be harnessed rather than fought against to surpass the diffraction-limit [4.5], to obtain temporal control by means of spatial manipulation only 6.77 , or, vice versa, to achieve spatial control by temporal manipulation 8, 9. Related to these results, it was long ago suggested by Freund 10] that a characterized random medium can be used as an arbitrary optical component. Indeed, it was recently shown that a random medium can be used as a lens $1,5,11,12$, a mirror [13, a spectralpolarimetric analyzer 14, a pulse shaping device 6, 6] and an arbitrary waveplate 15. Here we demonstrate the transformation of the random medium into an arbitrary spectral filter by wavefront shaping.

When an ultrashort pulse is scattered in a random medium, it undergoes both spatial and temporal distortions. As result of the spatio-temporal coupling in the random medium, both the spatial and temporal distortions can be corrected by manipulating the spatial shape of the incident wavefront 6, 7]. Since the scattering medium couples the spatial and temporal degrees of freedom, it also couples the spatial and spectral degrees of freedom. Here we exploit the same coupling to control the spectral profile of a broadband source (not necessarily an ultrashort pulsed source) focused through the random medium by manipulating its wavefront. We show that this technique can be used to generate arbitrary optical filters at a controlled focus.

To implement these ideas, a phase-only spatial light modulator (SLM) is used to shape the incident wavefront. The light passing through each of the SLM pixels generates a different temporal speckle pattern after propagating through the scattering medium (Fig. 19). These different random temporal patterns form an effective temporal basis for temporal control. Consequently, as each temporal patten is related to a particular spectral pattern by Fourier relation, it provides an effective spectral basis (Fig. 1b) which can be used to achieve spectral control, with a resolution determined by the interaction time of the light in the medium.

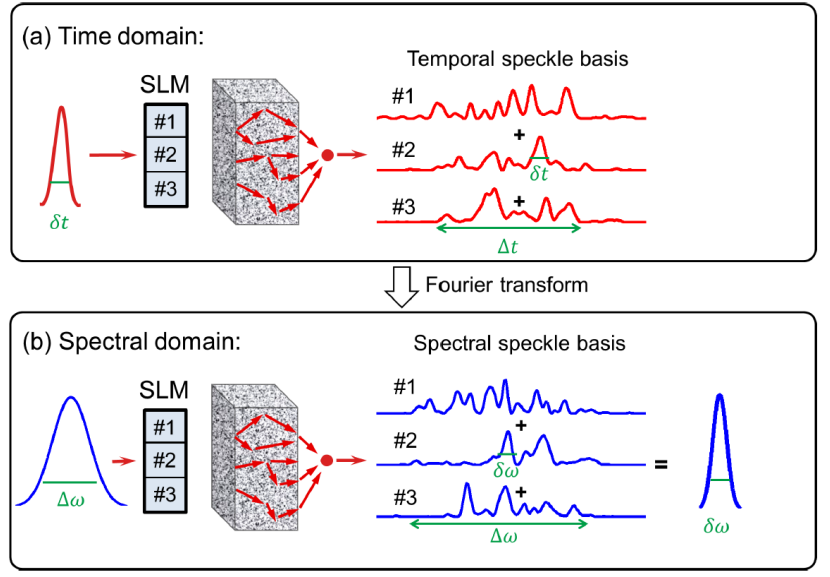

Fig. 1. Scattering in a random medium couples each SLM pixel to a different temporal (and spectral) pattern when measured at a particular output point, thus forming a random effective basis for temporal/spectral control by the SLM. In the temporal domain (a) an ultrashort pulse with duration of $\delta t$ at the input is scattered into different temporal patterns characterized by the same minimal feature size $\delta t$ and a temporal span $\Delta t$ determined by the interaction time of the light inside the medium. In the spectral domain (b) a broadband input with a bandwidth of $\Delta \omega \propto \frac{1}{\delta t}$ is scattered into different spectral patterns, characterized by a minimal feature size $\delta \omega \propto \frac{1}{\Delta t}$ and spanning the full $\Delta \omega$ of the source. A phase only SLM at the input can thus control the coherent interference of these random spectral patterns, generating an arbitrary optimized spectral pattern at the output.

Schematic sketches of these temporal and spectral 
speckle patterns are presented in Fig,1. The temporal patterns plotted in Fig,1(a) are characterized by two properties: the shortest temporal feature, or "temporal speckle" of characteristic duration $\delta t$, and the total temporal span $\Delta t . \delta t$ is determined by the bandwidth of the broadband laser source $\delta t \propto \frac{1}{\Delta \omega}$, while the temporal span $\Delta t$ is determined by the interaction time of the light in the scattering medium, and by optical path length differences when using an off-axis configuration (when considering the spectrum of a spot which is not on the optical axis) 16. The spectral characteristics of the speckle patterns presented in Fig, 1(b) are related to the temporal patterns by Fourier transform: The spectral span $\Delta \omega$ is simply the bandwidth of the broadband source which is inversely proportional to the temporal speckle width $\Delta \omega \propto 1 / \delta t$, while the narrowest spectral feature ("spectral speckle") $\delta \omega$ is dictated by the total temporal span $\delta \omega \propto 1 / \Delta t$. In terms of wavelength the minimal speckle size is given by:

$$
\delta \lambda=\frac{\lambda_{0}^{2}}{c \Delta t}
$$

where $\lambda_{0}$ is the central wavelength of the broadband source and $c$ is the speed of light. The spectral speckle width, $\delta \lambda$, dictates the spectral resolution of the random medium as a spectral filter or as a pulse shaping device 6]. While for a standard grating-based spectrometer the resolution is determined by the number of grating lines illuminated by the beam, here the resolution is determined by $\delta \lambda$ which is a property of the scattering medium and the geometry. It is not hard to see that, perhaps counter intuitively, a more scattering sample yields higher spectral resolution.

As in other utilizations of wavefront-shaping [1], the control is obtained by optimizing the phases of the SLM elements to minimize a cost function, which in this case is defined as the difference from a desired spectral function at the spatial measurement position. We note that only a small fraction of the incident energy is actually involved in the process.

The experimental setup for spectral control through a random medium by wavefront-shaping is shown in Fig, 2 . The simple experimental setup consists of a spatiallycoherent broadband laser source (a femtosecond laser Idesta Octavius-1G), a phase only 2-dimensional SLM (Hamamatsu model X10468-01), a focusing lens, a scattering medium (Polycarbonate diffuser) and a fibercoupled spectrum analyzer (Ando model AQ-6315A). We use the SLM to shape the wavefront of the incident field which is then focused with the lens on the scattering medium (note that the lens is not required to achieve spectral control, and is only used in order to increase the measured intensities). The scattered light at a single point behind the medium is coupled to the optical spectrum analyzer by a multimode fiber which is placed $1 \mathrm{~cm}$ from the scattering medium. For optimal spectral contrast, a single spatial speckle should be coupled to the fiber. The experiment takes place by optimizing the SLM

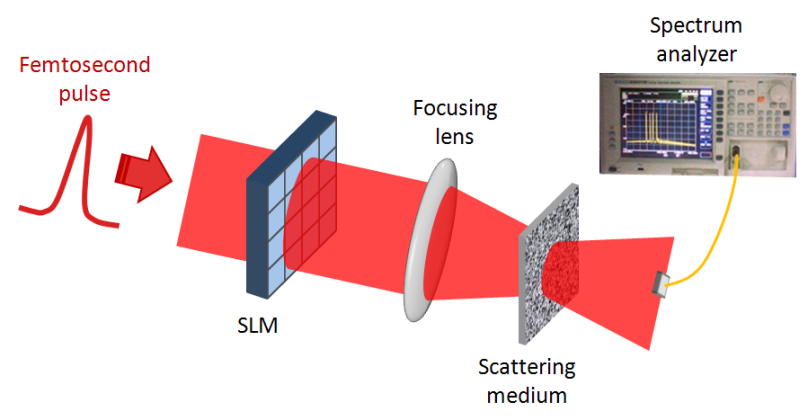

Fig. 2. Experimental setup for spectral control of a broadband source through a random medium by wavefront shaping. An SLM controls the wavefront of the incident spatially-coherent broadband field (not necessarily an ultrashort pulse). The wavefront shaped field is focused on the scattering medium with a lens. The scattered light at a particular spatial position behind the medium is measured by an optical spectrum analyzer, which generates a feedback signal for optimizing the incident wavefront to attain the desired spectral pattern.

phase-pattern using a genetic algorithm 17 to yield the desired spectral shape on the optical spectrum analyzer.

It is important to notice that since the feedback signal is the shape of the spectrum measured by the spectrum analyzer, the experiment is insensitive to the spectral phases. Therefore, the requirements from the source used for spectral control are only spatial coherence and broad spectrum, and it does not have to be a temporally coherent laser source (such as the ultrashort laser source used in our experiments). This means that any spatially coherent broadband source such as super-luminous diodes, super-continuum sources, or spatially-filtered thermal sources could be used as well.

To demonstrate the narrowest spectral filter this method allows, we simply choose one spectral feature in the measured scattered spectrum presented in Fig 3 (a) and maximize its power. The result of this optimization is shown in Fig, 3(b) where the blue line shows the initial spectrum and the red line shows the resulting spectrum after optimization. The inset of Fig $3(\mathrm{~b})$ is the SLM patten that yields the optimal result. The full width half maximum (FWHM) of the optimized spectrum is $5.5 \mathrm{~nm}$ (spectrometer resolution is $0.1 \mathrm{~nm}$ ) and the intensity enhancement comparing to the average unoptimized power is approximately 50. From this FWHM and with the help of Eq1, the scattering interaction time can be estimated to be $\Delta t=400 f s$.

In order to demonstrate more complex filters, we present the results of a dual bandpass filter in Fig]3(c). Here we choose two different spectral speckles and maximize their mutual power. This procedure results in two separated narrow spectral transmission bands. The FWHM of each one of the two lobes is the same as for the single lobe. However, similar to spatial focusing experiments 11, the enhancement of each of the two lobes compared to the background is half of the enhancement 

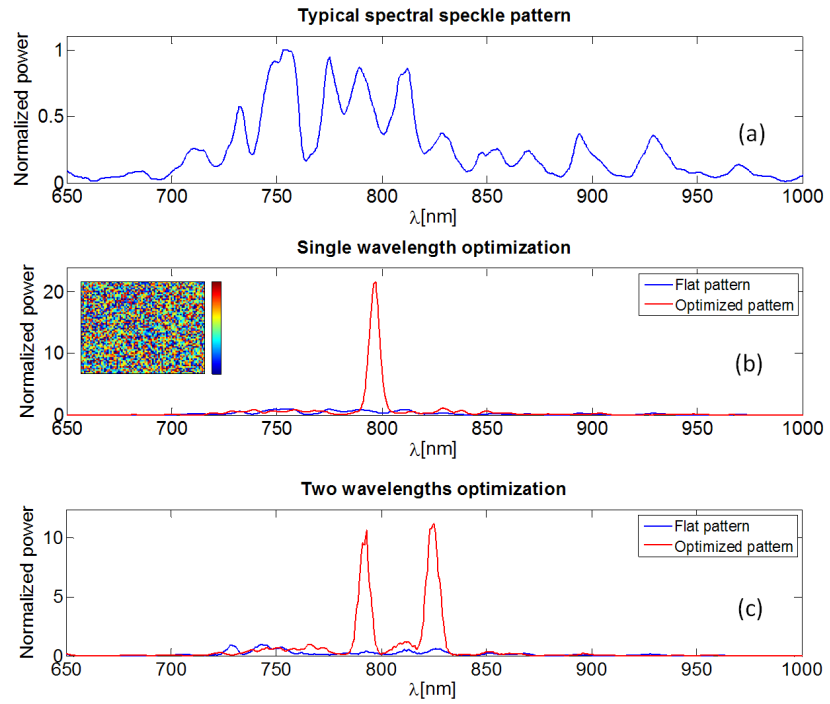

Fig. 3. Experimental results: (a) unoptimized spectrum of the scattered broadband source at the measurement point. (b) Optimized (red line) and unoptimized (blue line) spectra for a narrow bandpass filter optimization (inset: SLM optimized phase pattern, the maximum phase is $2.3 \pi$ ). (c) Optimized (red line) and unoptimized (blue line) spectra for a dual bandpass filter optimization. All spectral powers are normalized to the peak power of the unoptimized spectrum.

achieved for a single band. This contrast is determined by the number of degrees of control 1, and is the limit for the complexity of the controlled filter.

In summary, we have shown that random scattering can be exploited for spatial and spectral control of a broadband source inside/through a random medium by wavefront shaping. This technique can generate an arbitrary spectral filter at a specific spatial position. One can envisage using this tool for controlled spectroscopy inside a random-medium [18, or for spectrally resolved imaging or information transmission 12 through a random medium. Different spatial output points can be simultaneously optimized to different spectral filters using a single phase pattern. This can enable transformation of a random medium into a grating 10 or an optimized spectrum analyzer. Interestingly, in analogy to the spatial "memory effect" in thin scattering media [19], performing our experiment using a thin scattering medium and an off-axis geometry will result in spectrally scaling the single-point optimized spectral function when the distance of the measurement point from the optical axis is varied, using the same SLM phase-pattern. This is the result of the correlation between speckle patterns at different wavelengths in a thin scattering medium (e.g. in surface scattering) 16. Optimizing a single wavelength at a single point would thus lead to dispersing the full spectrum wavelength-by-wavelength in the radial direction.

E.S. is supported by the Adams Fellowship Program of the Israel Academy of Sciences and Humanities. This work was supported also by MINERVA and the ERC grant QUAMI.

\section{References}

1. I.M. Vellekoop, and A.P. Mosk, Opt. Lett. 32, 2309-2311 (2007).

2. Z. Yaqoob, D. Psaltis, M.S. Feld, and C. Yang, Nat. Photon. 2, 110-115 (2008).

3. M. Fink, Phys. Today 50, 34-40 (1997).

4. G. Lerosey, J. de Rosny, A. Tourin, and M. Fink, Science 315, 1120-1122 (2007).

5. I.M. Vellekoop, A. Lagendijk, and A.P. Mosk, Nat. Phot. 4, 320-322 (2010).

6. O. Katz, E. Small, Y. Bromberg, and Y. Silberberg, Nat. Phot. 5, 372-377 (2011).

7. J. Aulbach, B. Gjonaj, P. M. Johnson, A. P. Mosk, and A. Lagendijk, Phys. Rev. Lett. 106, 103901 (2011).

8. D. J. McCabe, A. Tajalli, D.R. Austin, P. Bondareff, I. A. Walmsley, S. Gigan, and B. Chatel, Nat. Comm. 2, 447 (2011).

9. F. Lemoult, G. Lerosey, J. de Rosny, M. Fink, Phys. Rev. Lett. 103, 173902 (2009).

10. I. Freund, Physica A: Statistical Mechanics and its Applications 168, 49-65 (1990).

11. S. M. Popoff, G. Lerosey, R. Carminati, M. Fink, A. C. Boccara, and S. Gigan, Phys Rev Lett 104, 100601 (2010).

12. S. Popoff, G. Lerosey, M. Fink, A. C. Boccara, and S. Gigan, doi:10 1038/ncomms1078 (2010).

13. O. Katz, E. Small, and Y. Silberberg, arXiv 1202.2078 (2012).

14. T. Kohlgraf-Owens, and A. Dogariu, Opt. Lett. 35, 2236-2238 (2010).

15. Y. Guan, O. Katz, E. Small, J. Zhou and Y. Silberberg, in preparation.

16. E. Small, O. Katz, and Y. Silberberg, Opt. Express 20 5189-5195 (2012).

17. D. B. Conkey, A. N. Brown, A. M. Caravaca-Aguirre, and R. Piestun, Opt. Express 20 4840-4849 (2012).

18. T. Svensson, E. Adolfsson, M. Lewander, C. T. Xu, and S. Svanberg, Phys. Rev. Lett. 107, 143901 (2011).

19. I. Freund, M.Rosenbluh, and S. Feng, Phys. Rev. Lett. 61, 2328-2331 (1988). 